\title{
The Thermodynamic Influence of Trapped Water Molecules on a Protein-Ligand Interaction**
}

\author{
Christian M. Stegmann, Daniel Seeliger, George M. Sheldrick, Bert L. de Groot, and \\ Markus C. Wahl*
}

The rational design of protein-binding substances requires an in-depth understanding of the energetics of protein-ligand interactions, including the free energy $(\Delta G)$, enthalpy $(\Delta H)$, entropy $(\Delta S)$, and the change of heat capacity at constant pressure $\left(\Delta C_{p}\right)$ upon binding. ${ }^{[1]}$ Previously, the $\Delta C_{p}$ of binding has been correlated with changes in the accessible polar and apolar surface areas of the interacting species, ${ }^{[2,3]}$ suggesting that hydrophobic interactions are the primary source of heat capacity effects (for a review, see reference [4]). However, these considerations leave discrepancies between calculated and observed $\Delta C_{p}$ values in a number of cases. ${ }^{[5]}$ In particular, the effects of solvent (typically water) and solutes (for example ions) buried upon complex formation are neglected; yet it is well known that these molecules can form discrete noncovalent bonds to both the protein and the ligand, thereby influencing recognition processes. For example, interface water molecules can act as shape modifiers, hydrogen bond bridges, or as electrostatic screens. ${ }^{[6]}$ Furthermore, solvent and solute molecules trapped upon a protein-ligand interaction experience a reduction in amplitude of their vibrational modes, thus giving rise to a change in the heat capacity. ${ }^{[7-9]}$ To

[*] C. M. Stegmann, Dr. D. Seeliger, Dr. B. L. de Groot, Prof. Dr. M. C. Wahl

Research Group X-ray Crystallography, Computational Biomolecular Dynamics Group, Max Planck Institute for Biophysical Chemistry Am Fassberg 11, 37077 Göttingen (Germany)

E-mail: mwahl@gwdg.de

Homepage: http://www.mpibpc.gwdg.de/groups/wahl/

Prof. Dr. M. C. Wahl

Freie Universität Berlin, Institut für Chemie und Biochemie, AG Strukturbiochemie

Takustrasse 6, 14195 Berlin (Germany)

Prof. Dr. G. M. Sheldrick

University of Göttingen, Department of Structural Chemistry Tammannstrasse 4, 37077 Göttingen (Germany)

[**; We are grateful to Gleb P. Bourenkov, Ehmke Pohl and Ina Dix for help with data collection, Ulrich Reidt for initial purification and crystallization trials of $\mathrm{CypG}_{\text {pplase }}$, Pawel Burkhardt for advice on ITC experiments, Kay Diederichs for providing multiprocessor versions of SHELXL, and Reinhard Lührmann for generous access to his facilities and continued interest. C.M.S. is a fellow of the German National Academic Foundation (Studienstiftung des Deutschen Volkes). This research was supported by a grant from the VW Foundation (VW I/81 649), by the German Research Foundation (DFG-GR 207914), and by the Max Planck Society. Atomic coordinates and structure factors have been deposited in the RCSB Protein Data Bank (www.rcsb.org) under PDB IDs 2wfi and 2wfj. Supporting information for this article is available on the WWWW under http://dx.doi.org/10.1002/anie.200900481. date, there is no experimental estimate for the size of this effect in a protein-ligand interaction.

Herein, we investigated the thermodynamic consequences of trapping water molecules upon interaction of human cyclophilin G (CypG; an enzyme with peptidyl prolyl cis/trans isomerase (PPIase) activity) with the immunosuppressive cyclic peptide cyclosporin A (CsA). Comparison of the CsA binding of the wild type (wt) CypG to a point mutant allowed us to single out the influence of trapped solvent molecules on the heat capacity change while excluding a significant differential contribution from buried surface areas.

CypG contains an $\mathrm{N}$-terminal cyclophilin domain (CypG PPIase $_{\text {; }}$ residues 1-177) that binds CsA and a C-terminal Ser-Arg dipeptide-rich (RS) domain (residues 178-754). ${ }^{[10,11]}$ We determined ab initio crystal structures at $0.75 \AA$ resolution of $\mathrm{CypG}_{\text {PPIase }}$ alone and in complex with CsA. $\mathrm{CypG}_{\text {PPIase }}$ binds CsA in a similar manner as described for $\mathrm{CypA}^{[12,13]}$ (Figure 1a,b), without undergoing significant conformational changes (root-mean-square deviation of $0.20 \AA$ for $174 \mathrm{C} \alpha$
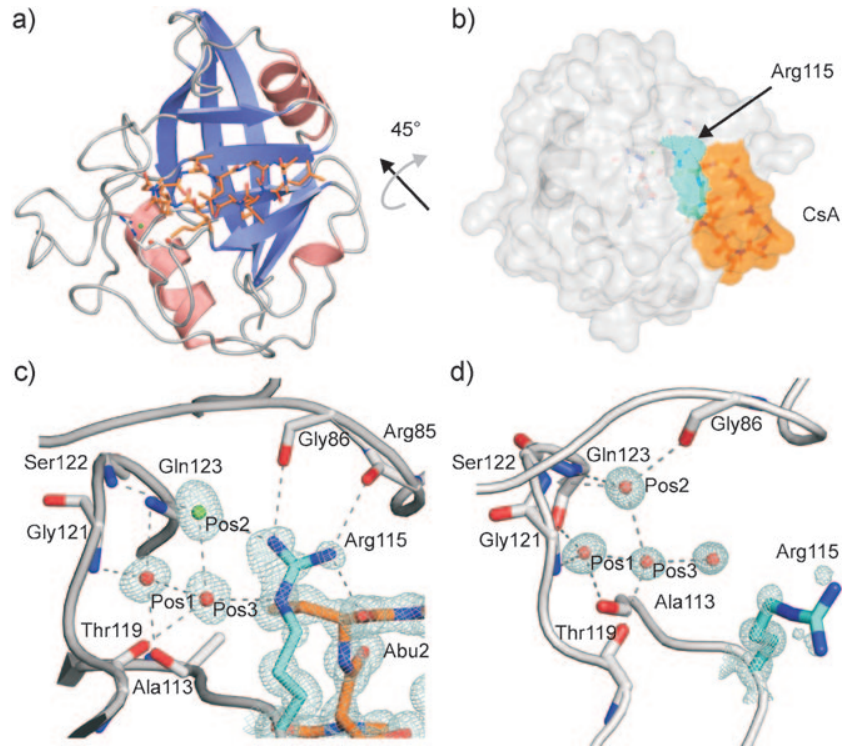

Figure 1. a) Crystal structure of $\mathrm{CypG}_{\text {pplase }}$ (ribbon plot) in complex with CsA (sticks, carbon: orange). b) The Arg115 side chain (cyan) adopts a closed conformation when complexed with CsA. c) In the CsA complex, the guanidinium group of Arg115 is well-ordered and hydrogen bonds to the carbonyl oxygen atoms of Arg85 and Gly86 and to the carbonyl oxygen of Abu2 of CsA, thereby burying two water molecules (red spheres; positions 1 and 3 ) and a chloride ion (green

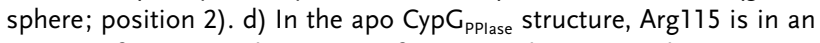
open conformation ( $d$, major conformation shown). Mesh: $\sigma_{\mathrm{A}^{-}}$ weighted electron densities contoured at the $1.5 \sigma$ level. 
atoms of apo and CsA-bound $\left.\mathrm{CypG}_{\text {PPIase }}\right)$. However, Arg115 in $\mathrm{CypG}_{\mathrm{PPI} \text { ase }}$ replaces an alanine at the corresponding site in CypA (see Supporting Information, Figure S1). Upon complex formation, the Arg115 side chain engages in direct hydrogen bonds to the carbonyl oxygen atom of Abu2 in CsA and also to the carbonyl oxygen atoms of Arg85 and Gly86 of $\mathrm{CypG}_{\text {PPIase }}($ Figure $1 \mathrm{c}$ ). These interactions are not available to the equivalent alanine in CypA. Arg115 thereby seals the socalled Abu pocket, ${ }^{[12]}$ in which two water molecules (at positions 1 and 3) and a chloride ion (at position 2) become trapped.

The identity of the chloride ion was confirmed by analysis of anomalous difference Fourier maps (Supporting Information, Figure S2). The two water molecules are tetrahedrally engaged in hydrogen bonds: the water at position 1 hydrogen bonds to the backbone amides of Gly121 and Gln123, to the backbone carbonyl of Ala113, and to the water at position 3 . The water at position 3 hydrogen bonds to the guanidinium group of Arg115, the carbonyl of Thr119, and the chloride in position 2. The chloride in turn is coordinated by the amide of Ser122 and the guanidinium group of Arg115 (Figure 1c). In the apo form, the Arg115 side chain is partially disordered (Figure 1d), leaving the Abu pocket open. Here, all three positions are occupied by water molecules. The position 1 water forms hydrogen bonds to the backbone $\mathrm{NH}$ group of Gly121, the carbonyl groups of Ala113 and Gln123, and the water at position 3 . The water at position 2 hydrogen bonds to the amides of Ser122 and Gln123, the carbonyl of Gly86, and the water at position 3 (Figure 1d). Thus, Arg115 seems to act like a lid that closes and is locked in place upon CsA binding. In CypA, the alanine corresponding to Arg115 cannot form a similar lid, presumably permitting free flux of solvent and solutes into and out of the cavity when CsA is bound.

As crystallography cannot distinguish between site occupancy and residence time, we performed molecular dynamics (MD) simulations of apo wt $\mathrm{CypG}_{\text {PPIase }}$, wt $\mathrm{CypG}_{\text {PPIase }}$ in complex with CsA, and a $\mathrm{CypG}_{\text {PPIase }}$ (Arg115Ala) point mutant in complex with CsA (ten independent simulations of $10 \mathrm{~ns}$ length for each system). The simulations revealed that a chloride ion at position 2 in the $\mathrm{CypG}_{\text {PPIase }} / \mathrm{CsA}$ complex can be exchanged with water and that chloride occupied that site owing to its high concentration (400 mM) in the crystallization setup. At a near-physiological chloride concentration $(150 \mathrm{~mm})$, the chloride ion was replaced by a water molecule in five out of ten simulations within the simulation time of $10 \mathrm{~ns}$, whereas the other two water molecules at positions 1 and 3 remained close to the crystallographic configuration. The water in position 2 engages in alternative bonding schemes to the position 3 water and either to Arg115 (Figure 2a) or to the carbonyl of Gly85 (not shown). Thus, at close to physiological conditions, water and chloride bind with similar occupancy at position 2 .

In agreement with the crystal structures, the MD simulations revealed a significant decrease in the flexibility of Arg115 upon CsA binding. Although Arg115 exhibits similar rates of dihedral transitions at its $\chi 1$ angle in apo $\mathrm{CypG}_{\text {PPIase }}$ and in $\mathrm{CypG}_{\text {PPIase }} / \mathrm{CsA}$, the rates of transitions at $\chi 2, \chi 3$, and $\chi 4$ are significantly reduced in the complex (Figure $2 b$ ). When Arg115 adopts an elongated conformation and forms hydro- a)

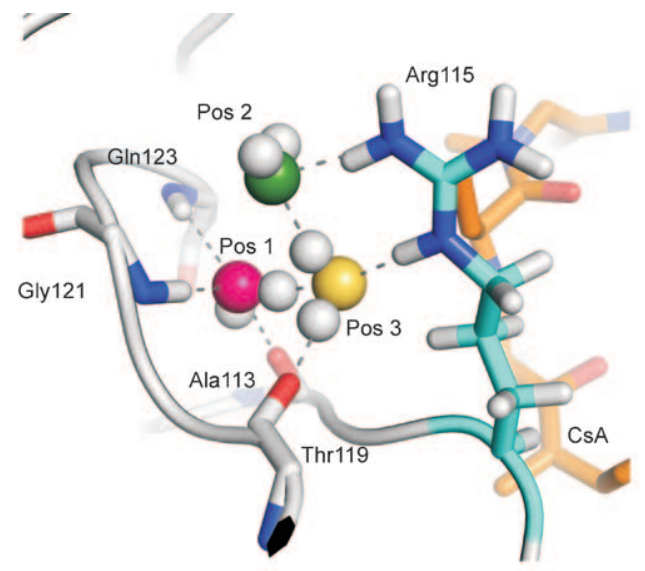

b)

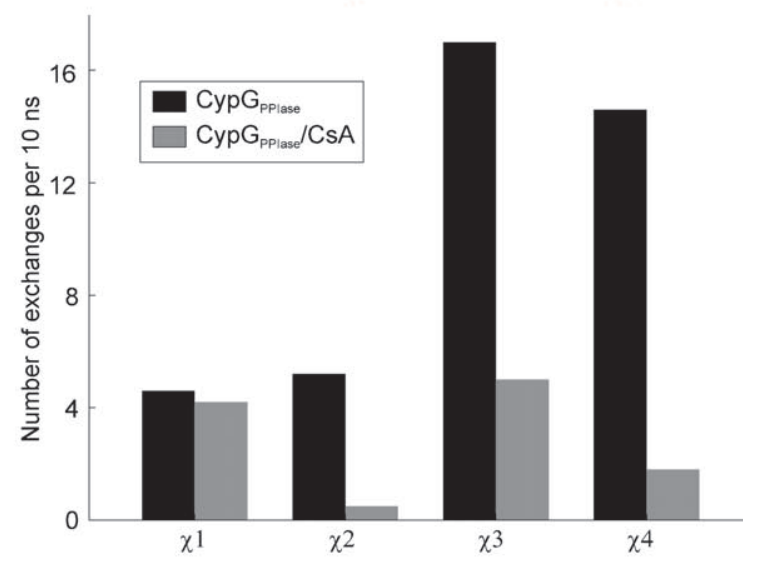

c)

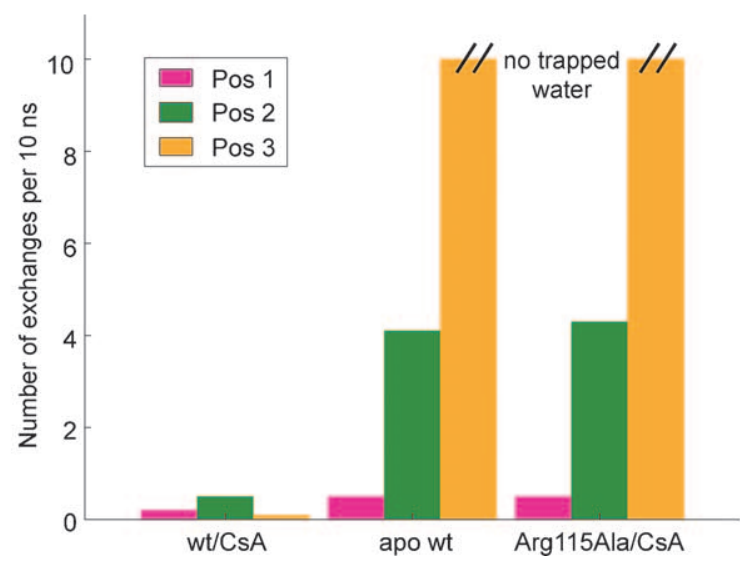

Figure 2. a) Representative locations and hydrogen bonding of trapped water molecules at positions 1, 2, and 3 during a simulation of the $\mathrm{CypG}_{\mathrm{pPlase}} / \mathrm{CsA}$ complex. Dashed lines indicate hydrogen bonds. b) Histogram of dihedral angle transitions of $\operatorname{Arg} 115$ in apo wt CypG pplase (black bars) and in the $\mathrm{CypG}_{\text {pplase }} / \mathrm{CsA}$ complex (gray bars). The flexibility of the side chain is significantly reduced upon binding of CsA. c) Residence times of water molecules at the three binding sites in the Abu pocket. Whereas the water at position 1 does not exchange in any of the systems, the water molecules at positions 2 and 3 show increased exchange rates upon mutation of Arg 115 to Ala (i.e. removal of the "lid" of the Abu pocket) and in the absence of CsA (i.e. upon "unlocking" the lid).

gen bonds with the carbonyl oxygen atoms of Arg85 or Gly86, a cavity is formed in which water molecules become trapped (Figure 2a; Supporting Information, Figure S3). Owing to the 
reduced flexibility of Arg115 in the $\mathrm{CypG}_{\text {PPIase }} / \mathrm{CsA}$ complex, the lifetime of this conformation is increased and, as a consequence, the residence times of two of the three water molecules in the cavity are significantly prolonged (Table 1 ; Supporting Information, Figure S3).

Table 1: Exchanges per $10 \mathrm{~ns}$ at positions in the Abu pocket.

\begin{tabular}{lllc}
\hline Simulation & Pos 1 & Pos 2 & Pos 3 \\
\hline wt CypG & 0.2 & 0.5 & 0.1 \\
apo wt CypG $/$ CsA & 0.5 & 4.1 & $>10$ \\
CypG $_{\text {PPlase }}$ (Arg115Ala)/CsA & 0.5 & 4.3 & $>10$ \\
\hline
\end{tabular}

The exchange rate of water molecules at position 1 is very low in all systems and in all simulation runs $(0.2$ exchanges per $10 \mathrm{~ns}$ in the wild-type complex; 0.5 exchanges per $10 \mathrm{~ns}$ in apo wt $\mathrm{CypG}_{\text {PPIase }}$ and the mutant complex; Figure 2c). Although the residence times of the position 1 water molecules in wt $\mathrm{CypG}_{\text {PPIase }} / \mathrm{CsA}$ simulations appear to be longer than in the other simulations (Figure 2c), the absolute numbers of exchanges are too low to accurately calculate differences in the exchange rates. Longer simulations of the three systems revealed that a water molecule can remain at position 1 for up to $180 \mathrm{~ns}$.

In contrast, the exchange rate at position 2 is strongly dependent on both CsA binding and the Arg115Ala mutation. Whereas the exchange rate at position 2 is also very low in the wt $\mathrm{CypG}_{\text {PPIase }} / \mathrm{CsA}$ complex (0.5 exchanges per $\left.10 \mathrm{~ns}\right)$, it increases eightfold in the apo form (to 4.1 exchanges per $10 \mathrm{~ns}$; Figure $2 \mathrm{c}$ ). A comparable increase in the exchange rate (to 4.3 exchanges per $10 \mathrm{~ns}$ ) is observed in the simulations of the mutant $\mathrm{CypG}_{\text {PPIase }}($ Arg115Ala)/CsA complex (Figure 2c).

Similarly, in the wt $\mathrm{CypG}_{\text {PPIase }} / \mathrm{CsA}$ complex, a water molecule at position 3 has a very low exchange rate (0.1 exchanges per $10 \mathrm{~ns}$ ). In the apo $\mathrm{CypG}_{\text {PPIase }}$ simulations, in contrast, we did not observe a prolonged residence time of water molecules at this position (Figure $2 \mathrm{c}$ ). Furthermore, no water was trapped at this position in the simulations of the mutant $\mathrm{CypG}_{\text {PPIase }}$ (Arg115Ala)/CsA complex. Therefore, our simulations show that the reduced flexibility of Arg115 in the wt $\mathrm{CypG}_{\mathrm{PPIase}} / \mathrm{CsA}$ complex has a major effect on the residence times of two of the three trapped water molecules in $\mathrm{CypG}_{\mathrm{PPI} I \mathrm{se}} / \mathrm{CsA}$, whereas the water molecule in position 1 appears to be structurally relevant and is neither affected by CsA binding nor by mutation of Arg115.

The above analyses suggest that an arginine instead of an alanine at position 115 contributes negatively to the $\Delta C_{p}$ of the complex formation with CsA by mediating the trapping of water molecules. To test this hypothesis, we performed calorimetric titrations at a range of temperatures with wt $\mathrm{CypG}_{\text {PPIase }}$ and the $\mathrm{CypG}_{\text {PPIase }}$ (Arg115Ala) point mutant. At $25^{\circ} \mathrm{C}$, CsA binding to wt and mutant $\mathrm{CypG}_{\text {PPIase }}$ has a similar binding free energy owing to enthalpy/entropy compensation effects (Table 2). However, a plot of the interaction enthalpies versus temperature reveals that the $\Delta C_{p}$ of binding of CsA to $\mathrm{CypG}_{\mathrm{PPIase}}$ decreases by $114 \mathrm{cal} \mathrm{mol}^{-1} \mathrm{~K}^{-1}$ compared to the $\Delta C_{p}$ of binding of CsA to the mutant (Figure 3).
Table 2: Thermodynamic data from isothermal titration calorimetry.

\begin{tabular}{lcc}
\hline Titrant & CypG $_{\text {PPlase }}$ & CypG $_{\text {pPlase }}$ (Arg115Ala) \\
\hline$T\left[{ }^{\circ} \mathrm{C}\right]$ & 25 & 25 \\
$K_{\mathrm{D}}[\mathrm{nM}]$ & 70 & 64 \\
$\Delta H_{\text {obs }}\left[\mathrm{cal} \mathrm{mol}^{-1}\right]$ & $-12790 \pm 73$ & $-13360 \pm 81$ \\
$\Delta S\left[\mathrm{cal} \mathrm{mol} \mathrm{K}^{-1}\right]$ & -10.2 & -11.9 \\
$-T \Delta S\left[\mathrm{cal} \mathrm{mol}^{-1}\right]$ & 3039 & 3546 \\
$\Delta G\left[\mathrm{cal} \mathrm{mol}^{-1}\right]$ & -9750 & -9813 \\
$\Delta C_{p}\left[\mathrm{cal} \mathrm{mol}^{-1} \mathrm{~K}^{-1}\right]$ & $-580 \pm 31$ & $-466 \pm 11$ \\
\hline
\end{tabular}

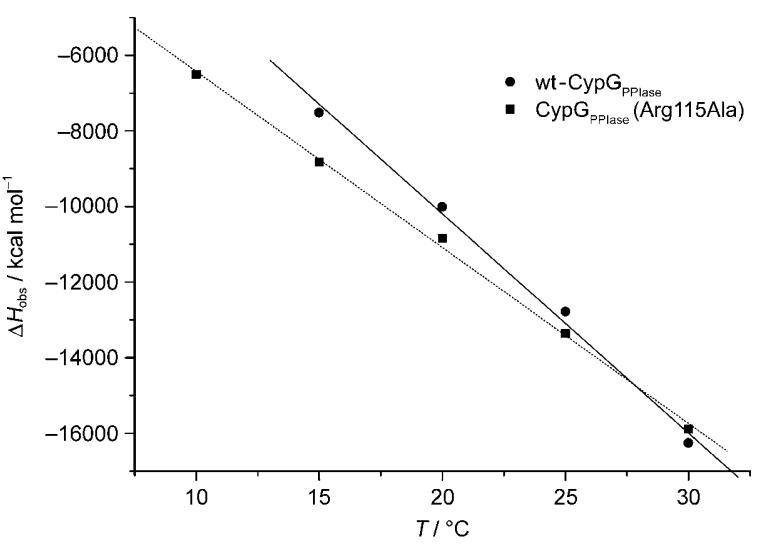

Figure 3. Integrated enthalpies of calorimetric titration experiments $\left(\Delta H_{\text {obs }}\right)$ plotted against the temperature $(T)$ for the wt $C_{\text {pyplase }} / \mathrm{CsA}$ and the $\mathrm{CypG}_{\text {pplase }}$ (Arg115Ala)/CsA interaction. The differential change in heat capacity $\left(\Delta C_{p}\right)$ upon CsA binding is given by the slopes of the curves.

The protein surface areas buried upon CsA binding are $426 \AA^{2}$ in wt CypG $_{\text {PPIase }}$ and $444 \AA^{2}$ in CypG $_{\text {PPIase }}$ (Arg115Ala) (model based on the wt crystal structure). On the basis of changes in the accessible polar and apolar surface areas alone ${ }^{[3]}$ this increase in buried surface area would result in an increase in $\Delta C_{p}$ of binding. Instead, we observe a decrease in $\Delta C_{p}$ of binding, which we attribute to the differential trapping of two water molecules in the wt complex. Although the exchange rates of the water molecules at positions 2 and 3 increase to different extents upon removal of the Arg115 side chain (Figure 2c), the current statistics do not allow us to quantify individual contributions to $\Delta C_{p}$ of binding. Our results, therefore, provide an experimental value of $(-57 \pm$ 21) $\mathrm{cal} \mathrm{mol}^{-1} \mathrm{~K}^{-1}$ for the average heat capacity change owing to the sequestration of one water molecule upon formation of a protein-ligand complex. This experimental estimate agrees very well with theoretical figures and studies of model compounds. Holdgate and co-workers ${ }^{[14]}$ suggested a value of $(-48 \pm 31)$ cal mol $^{-1} \mathrm{~K}^{-1}$. Cooper ${ }^{[5]}$ calculated a change in $\Delta C_{p}$ of at least $-18 \mathrm{cal} \mathrm{mol}^{-1} \mathrm{~K}^{-1}$ per sequestered water molecule based on simple lattice models. Habermann and Murphy ${ }^{[15]}$ determined a value of $(-60 \pm 8) \mathrm{cal} \mathrm{mol}^{-1} \mathrm{~K}^{-1}$ per sequestered water molecule based on the dissolution of $\mathrm{c}(\mathrm{AS})$ diketopiperazine crystals (a dipeptide model compound containing one water molecule in its crystalline form).

Taken together, our findings demonstrate that one amino acid substitution in the active site of a well-conserved enzyme can cause the burial of solvent molecules and thereby elicit a 


\section{Communications}

change in the thermodynamic signature of the interaction with ligands.

Received: January 25, 2009

Revised: March 18, 2009

Published online: June 4, 2009

Keywords: biophysics · drug design · isomerases .

molecular dynamics · structural biology

[1] R. Perozzo, G. Folkers, L. Scapozza, J. Recept. Signal Transduction Res. 2004, 24, 1.

[2] K. P. Murphy, D. Xie, K. C. Garcia, L. M. Amzel, E. Freire, Proteins Struct. Funct. Genet. 1993, 15, 113.

[3] R. S. Spolar, J. R. Livingstone, M. T. Record, Jr., Biochemistry 1992, 31, 3947.

[4] N. V. Prabhu, K. A. Sharp, Annu. Rev. Phys. Chem. 2005, 56, 521.

[5] A. Cooper, Biophys. Chem. 2005, 115, 89.
[6] C. S. Poornima, P. M. Dean, J. Comput.-Aided Mol. Des. 1995, 9, 500 .

[7] J. M. Sturtevant, Proc. Natl. Acad. Sci. USA 1977, 74, 2236.

[8] J. E. Ladbury, J. G. Wright, J. M. Sturtevant, P. B. Sigler, J. Mol. Biol. 1994, 238, 669.

[9] S. Bergqvist, M. A. Williams, R. O’Brien, J. E. Ladbury, J. Mol. Biol. 2004, 336, 829.

[10] L. Cavarec, T. Kamphausen, B. Dubourg, I. Callebaut, F. Lemeunier, D. Metivier, J. Feunteun, G. Fischer, N. Modjtahedi, J. Biol. Chem. 2002, 277, 41171.

[11] F. P. Nestel, K. Colwill, S. Harper, T. Pawson, S. K. Anderson, Gene 1996, 180, 151.

[12] V. Mikol, J. Kallen, G. Pflugl, M. D. Walkinshaw, J. Mol. Biol. $1993,234,1119$

[13] G. Pflügl, J. Kallen, T. Schirmer, J. N. Jansonius, M. G. Zurini, M. D. Walkinshaw, Nature 1993, 361, 91.

[14] G. A. Holdgate, A. Tunnicliffe, W. H. Ward, S. A. Weston, G. Rosenbrock, P. T. Barth, I. W. Taylor, R. A. Pauptit, D. Timms, Biochemistry 1997, 36, 9663.

[15] S. M. Habermann, K. P. Murphy, Protein Sci. 1996, 5, 1229. 